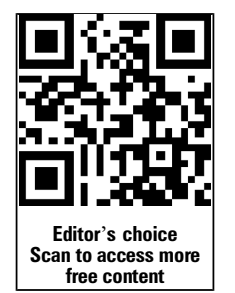

can to access mor ree content

${ }^{1}$ Department of Sports Medicine, Norwegian School of Sports Sciences, Oslo, Norway ${ }^{2}$ Oslo Center of Biostatistics and Epidemiology, Research Support Services, Oslo University Hospital, Oslo, Norway

Correspondence to Professor Ingar Holme; Department of Sports Medicine, Norwegian School of Sport Sciences, P.O. Box 4014 Ullevål Stadion, Oslo 0806, Norway; ingar.holme@nih.no

Accepted 27 March 2015

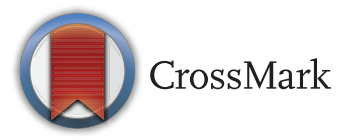

To cite: Holme I, Anderssen SA. Br J Sports Med 2015:49:743-748.

\title{
Increases in physical activity is as important as smoking cessation for reduction in total mortality in elderly men: 12 years of follow-up of the Oslo II study
}

\author{
I Holme, ${ }^{1,2}$ S A Anderssen ${ }^{1}$
}

\begin{abstract}
Background Physical activity (PA) at leisure by the elderly, and its relationship to cardiovascular (CV) and non-CV mortality, with and without competing risk, has been scarcely described. We determined the relationships between PA, smoking and 12-year CV, non-CV and allcause mortality in elderly Oslo men screened for CV disease in 1972-1973 and 2000.
\end{abstract}

Methods Among 14846 men born during 19231932 and participating in 1972-1973, there were 5738 participants in 2000. During the 12 years follow-up 2154 died. Cox regression modelling of mortality endpoints, with and without competing risk, was applied analysing PA variables hours per week of light or vigorous PA intensity and degree of PA at leisure. Comparisons of predictive ability between PA and smoking were done by receiver operating characteristics. Results Thirty minutes of PA per 6 days a week was associated with about $40 \%$ mortality risk reduction. There was a 5 years increased lifetime when comparing sedentary and moderate to vigorous physically active men. Associations to CV or non-CV mortality were slightly weakened, allowing competing risk. Conditional on the prevalence of smoking and PA, the degree of PA at leisure was almost as predictive as smoking with regard to the effects on mortality. Increase in PA was as beneficial as smoking cessation in reducing mortality.

Conclusions Even at the age of 73 years, PA is associated highly with mortality between groups of sedentary and active persons. Allowing for competing risk did not weaken these associations markedly. Public health strategies in elderly men should include efforts to increase PA in line with efforts to reduce smoking behaviour.

\section{INTRODUCTION}

Many studies have shown associations between degree of physical activity at leisure and mortality, cardiovascular $(\mathrm{CV})$ as well as non-CV. ${ }^{1-3}$ Relative mortality risk has often been reported to be $30-60 \%$ lower in those physically active as compared with sedentary persons. ${ }^{4}$ Such associations are also found in the elderly age group. ${ }^{6}$ Even if the degree of activity was only light, mortality risk was lower than that for sedentary participants. ${ }^{7}$

Comparisons of predictive power between physical activity and other risk factors for morbidity and mortality have been reported, but its results would depend heavily on how physical activity was defined and what measures of predictive ability were used. ${ }^{8}$ When analysing $\mathrm{CV}$ mortality in elderly participants, degree of competing risk will become substantial if follow-up is extended over longer periods of time. Ordinary Cox regression models will censor non-CV deaths and thus, shorten follow-up time with regard to CV mortality. Few reports of physical activity versus mortality have taken this into account.

The Oslo I CV study took place in 1972-1973, when all men aged $40-49$ years were invited to a screening examination. ${ }^{9} 10$ In year 2000, these attending men were invited again to an extended screening examination of $\mathrm{CV}$ health, called Oslo II. $^{11}$ Mortality of those who participated at both screenings was followed with respect to $\mathrm{CV}$ and non-CV mortality until 31 December 2011. The major exposure variables were degree of physical activity at leisure, hours per week of light and vigorous intensity physical activity.

The purposes of this study were: to estimate the relationships between exposure variables and 12-year CV, non-CV and all-cause mortality in elderly men during the first decade of the 21st century; to compare estimates with those derived from competing risk Cox regression models and finally, to compare relative risks of 28-year changes of the different activity exposure variables with that of changes in smoking habits.

\section{PARTICIPANTS AND METHODS}

The Oslo study has been described in detail elsewhere. ${ }^{9}{ }^{10}$ In short, 25915 men born in 19231932 were invited to a screening examination in 1972-1973 and 16203 participated (63\%). Conventional risk factors included non-fasting total cholesterol, systolic and diastolic blood pressure, and cigarette smoking. In addition, body height and weight were measured. The so-called Gothenburg questionnaire regarding degree of physical activity at leisure were elicited ${ }^{12}$ and the question has been well validated ${ }^{13}$ (sedentary: reading, watching television or other sedentary occupation; light activity: walking, bicycling or other forms of physical activity including walking or bicycling to and from working place, and Sunday walk for at least $4 \mathrm{~h}$ a week; moderate activity: exercise, sports, heavy gardening, etc, for at least $4 \mathrm{~h}$ /week; vigorous activity: hard training or competitive sports regularly several times a week). Men with a history of previous CV diseases, treated hypertension or diabetes were excluded from the analyses as well as men with missing values. This left 14846 participants in Oslo I. 
In year 2000, the surviving Oslo I study men $(n=12764)$ were invited to the Oslo II screening examination where the same variables and methods were applied. There were 6014 attending participants (47\%). However, other variables and characteristics were also measured, such as hours per week of light and vigorous intensity of physical activity (time spent on physical activity per week: none, $<1,1-2,3+$ hours), and history of previous diseases such as diabetes, myocardial infarction, stroke, length of education and high-density cholesterol. ${ }^{11}$ In some prediction analyses, we calculated an activity intensity variable consisting of nine categories, by combining the $4 \times 4$ matrix of light and vigorous intensity categories. The two intermediate categories (2 and 3 ) were collapsed for each variable. Analysis of CV (ischaemic heart disease, heart failure and stroke), non-CV and all-cause mortality were confined to the period from screening examination early in year 2000 to end of year 2011, almost 12 years of follow-up. In this period, 2154 deaths occurred among the 5738 included men who participated in Oslo I and in Oslo II.

This study was approved by a Regional Ethics Committee for scientific research in Norway, the Norwegian Data Inspectorate, and the Ministry of Health. The study complies with the Declaration of Helsinki.

\section{Statistical methods}

Cox regression models were used to compare the various outcomes according to levels of exposure variables (lowest level as reference), adjusted for age, smoking, diabetes, length of education, previous myocardial infarction and stroke. Exposure factor analysis for $\mathrm{CV}$ death, using non-CV death as a competing risk, was performed using the method of Fine and Gray. ${ }^{14}$ The predictive value of risk factors versus mortality was analysed by receiver operating characteristics (ROC) statistics. Model comparisons were made versus a full model with all selected risk factors.

Probability graphs of obtained ages by levels of activity for the three activity exposure factors, adjusted for risk factors, were made using STATA V.12. ${ }^{15}$

To check the assumptions of proportional hazards in the Cox regression models, time variant Cox regression models were run with time multiplied by exposure factor as the time-dependent covariate. For none of the end points or any exposure variable was there such a significant interaction term (data not given).

\section{RESULTS}

Table 1 gives an overview of the distributions of $\mathrm{CV}$, non-CV and all-cause deaths according to the levels of the exposure variables: time of light and vigorous physical activity per week, and Gothenburg question of physical activity degree at leisure. Also included in table 1 are the distributions of the adjustment factors. Degree of physical activity at leisure category 4 had few deaths and gave non-robust mortality results.

Table 1 Characteristics and descriptive statistics of exposure and adjustment factors for cardiovascular, non-cardiovascular and all-cause deaths

\begin{tabular}{|c|c|c|c|}
\hline \multicolumn{4}{|l|}{ Causes of death } \\
\hline Activity & Cardiovascular deaths (\%) & Non-cardiovascular deaths (\%) & All-cause deaths $(\%$ \\
\hline \multicolumn{4}{|l|}{ Light ( $\mathrm{N}=5452$ ) (hours) } \\
\hline None & $67(20.6)$ & $133(40.8)$ & $200(61.3)$ \\
\hline$<1$ & $59(15.6)$ & $123(32.7)$ & $182(48.4)$ \\
\hline $1-2$ & $163(10.6)$ & $434(28.3)$ & $597(39.0)$ \\
\hline $3+$ & $251(7.8)$ & $821(25.5)$ & $1072(33.3)$ \\
\hline \multicolumn{4}{|l|}{ Vigorous ( $\mathrm{N}=5287$ ) (hours) } \\
\hline None & $301(13.0)$ & $751(32.5)$ & $1052(45.5)$ \\
\hline$<1$ & $83(7.1)$ & $302(25.8)$ & $385(32.8)$ \\
\hline $1-2$ & $89(8.1)$ & $252(22.8)$ & $341(30.9)$ \\
\hline $3+$ & $41(5.9)$ & $146(21.0)$ & $187(26.9)$ \\
\hline \multicolumn{4}{|l|}{ Degree at leisure $(\mathrm{N}=4061)$} \\
\hline Sedentary & $96(13.3)$ & $272(37.8)$ & $368(51.1)$ \\
\hline Light & $209(8.1)$ & $598(23.1)$ & $807(31.2)$ \\
\hline Moderate & $45(6.4)$ & $120(17.0)$ & $165(23.4)$ \\
\hline Vigorous & $3(6.4)$ & $9(19.1)$ & $12(25.5)$ \\
\hline \multicolumn{4}{|l|}{ Smoking $(N=5704)$} \\
\hline None & $120(7.8)$ & $315(20.5)$ & $435(28.3)$ \\
\hline Previous & $327(1.3)$ & $859(27.2)$ & $1186(37.5)$ \\
\hline Current & $114(11.3)$ & $406(40.4)$ & $520(51.7)$ \\
\hline \multicolumn{4}{|l|}{ Diabetes $(N=5738)$} \\
\hline Yes & $71(18.5)$ & $134(34.9)$ & $205(53.4)$ \\
\hline No & $498(9.3)$ & $1455(27.2)$ & $1949(36.4)$ \\
\hline \multicolumn{4}{|l|}{ Myocardial infarction $(\mathrm{N}=5738)$} \\
\hline Yes & $158(23.0)$ & $194(28.2)$ & $352(51.2)$ \\
\hline No & $411(8.1)$ & $12395(27.7)$ & $1802(35.7)$ \\
\hline \multicolumn{4}{|l|}{ Cerebral stroke $(\mathrm{N}=5738)$} \\
\hline Yes & $97(22.7)$ & $148(34.6)$ & $245(57.2)$ \\
\hline No & $472(8.9)$ & $1441(27.2)$ & $1909(36.0)$ \\
\hline Age in 1972-1973 (Mean (SD)) ( $\mathrm{N}=5738)$ & $45.4(2.7)$ & $44.8(2.8)$ & $45.2(2.8)$ \\
\hline Education (years) (Mean (SD)) ( $\mathrm{N}=5522)$ & $11.6(3.7)$ & $11.7(3.8)$ & $11.7(3.8)$ \\
\hline
\end{tabular}


Concerning the three exposure activity variables there were negative associations to all outcome measures. Gradients measured by relative risks were slightly stronger for $\mathrm{CV}$ than non-CV mortality; however, for non-CV mortality too the gradients were substantial.

Adjusted analyses of the exposure activity variables versus the different causes of death are given in table 2. Irrespective of exposure factor for each outcome, the gradients were highly statistically as well as clinically significant. Less than $1 \mathrm{~h}$ of light activity per week was not sufficient to reach a statistically significant degree of risk reduction in any outcome, whereas higher amounts of light activity seemed to bring substantial associated risk reductions (32-56\%). For vigorous, on the contrary, less than $1 \mathrm{~h} /$ week was associated with $23-37 \%$ risk reduction, and highly significant for all end points. The lowest risk had the group with the longest intensity of vigorous intensity activity (36-49\% risk reductions). For degree of physical activity at leisure, mortality gradients were again strong, especially for non-CV and all-cause mortality $(38-57 \%$ risk reduction from sedentary to the intermediate level).

Figure $1 \mathrm{~A}-\mathrm{C}$ displays estimated probabilities for obtained age by levels of the three exposure variables. For all exposure factors, higher levels of activity were associated with 3-5 years extended lifetime (largest increase for Gothenburg question) as compared with lowest level, even in these elderly men.

To investigate whether changes in activity and smoking habits between the two screenings are associated with the subsequent mortality from year 2000, five categories of changes in activity and four categories of changes in smoking habits were created. For inactive men in Oslo I who had increased their degree of activity at Oslo II, the mortality rate was $44(26-57) \%$ lower as compared with sustained sedentary activity behaviour. Those who were at least intermediately active at Oslo I but sedentary at Oslo II had the same mortality as those who were sedentary at both occasions. Those who quit smoking between the Oslo I and II screenings had $31(18-41) \%$ lower mortality than those who smoked at both screening times.

For $\mathrm{CV}$, mortality there was a great degree of competing risk of non-CV mortality, but this was less so the other way around. The models applied above treated non-CV deaths as censored observations when CV mortality was analysed and vice versa when non-CV death was analysed. In table 3 relative risks are displayed for $\mathrm{CV}$ and non-CV death. Risk gradients were in the same direction as above but the strengths were somewhat weaker, though these still remained statistically significant in important cases judging from the CIs not covering 1.0. With respect to the two intensity questions, significance for $\mathrm{CV}$ death occurred for the highest intensity categories and for the Gothenburg question, in the intermediate category. Since degree of competing risk was less for non-CV as compared with $\mathrm{CV}$ mortality, risk gradients were less influenced by competing risk for non-CV as compared with CV death.

Table 4 gives ROC area values for $\mathrm{CV}$, non-CV and all-cause mortality for different models. The first line shows ROC areas when all factors are included. The second line gives the decrease in area $(0.013)$ when the two activity variables are included, but not smoking. When the Gothenburg question variable (line 4) is excluded but smoking included, the reduction amounts to 0.011 . When both activity variables are excluded but smoking included (line 5), the ROC area reduction is 0.019 . Thus, the ROC area contribution for activity seems to be about as large as that of smoking, over and above common adjustment factors.

\section{DISCUSSION}

Physical activity at leisure-as well as light and vigorous intensity activity-is associated powerfully and negatively to $\mathrm{CV}$, non-CV and all-cause mortality. The competing risk Cox regression model weakened the results, but the highest categories of the intensity questions and intermediate category of the Gothenburg question were still significant for CV death as compared with reference. The highest category of the Gothenburg question did not have sufficient power. For non-CV deaths, results were not much different as compared with total mortality due to the high percentage of such deaths.

Increased physical activity may introduce as much mortality reduction as smoking cessation in this age group.

A recent meta-analysis ${ }^{2}$ reported that an energy expenditure corresponding to $1000 \mathrm{Kcal} /$ week was associated with $20-30 \%$ reduced mortality, covering all ages and both genders. This amount is comparable to the moderate and intermediate degree

Table 2 Relative risk of cardiovascular, non-cardiovascular and all-cause mortality by physical activity, adjusted for potential confounders*

\begin{tabular}{|c|c|c|c|c|c|c|}
\hline \multicolumn{7}{|l|}{ Cause of death } \\
\hline Activity & $\begin{array}{l}\text { Cardiovascular } \\
\text { RR }(95 \% \mathrm{Cl})\end{array}$ & $\mathbf{P}$ & $\begin{array}{l}\text { Non-cardiovascular } \\
\text { RR }(95 \% \mathrm{CI})\end{array}$ & $\mathbf{P}$ & $\begin{array}{l}\text { All-cause } \\
\text { RR }(95 \% \mathrm{Cl})\end{array}$ & $\mathbf{P}$ \\
\hline Light intensity ( $\mathrm{N}=4662$ ) (hours) & & $<0.001$ & & $<0.001$ & & $<0.001$ \\
\hline None & 1 & & 1 & & 1 & \\
\hline$<1$ & $0.84(0.57$ to 1.23$)$ & 0.36 & 0.84 (0.64 to 1.09$)$ & 0.19 & 0.83 (0.67 to 1.04$)$ & 0.10 \\
\hline $1-2$ & 0.56 (0.41 to 0.77$)$ & $<0.001$ & 0.68 (0.55 to 0.84$)$ & $<0.001$ & 0.64 (0.53 to 0.76$)$ & $<0.001$ \\
\hline $3+$ & $0.44(0.32$ to 0.60$)$ & $<0.001$ & $0.62(0.50$ to 0.76$)$ & $<0.001$ & $0.56(0.47$ to 0.67$)$ & $<0.001$ \\
\hline Hard intensity ( $\mathrm{N}=4543$ ) (hours) & & $<0.001$ & & $<0.001$ & & $<0.001$ \\
\hline None & 1 & & 1 & & 1 & \\
\hline$<1$ & $0.63(0.49$ to 0.82$)$ & $<0.001$ & $0.77(0.67$ to 0.89$)$ & $<0.001$ & $0.74(0.65$ to 0.83$)$ & $<0.001$ \\
\hline $1-2$ & $0.69(0.53$ to 0.89$)$ & 0.005 & $0.72(0.62$ to 0.83$)$ & $<0.001$ & 0.71 (0.62 to 0.81$)$ & $<0.001$ \\
\hline $3+$ & $0.53(0.38$ to 0.75$)$ & $<0.001$ & 0.64 (0.53 to 0.78$)$ & $<0.001$ & 0.51 (0.52 to 0.72 ) & $<0.001$ \\
\hline Degree at leisure $(\mathrm{N}=3500)$ & & 0.036 & & $<0.001$ & & $<0.001$ \\
\hline Sedentary & 1 & & 1 & & 1 & \\
\hline Light & $0.72(0.55$ to 0.94$)$ & 0.017 & $0.58(0.50$ to 0.68$)$ & $<0.001$ & $0.62(0.54$ to 0.71$)$ & $<0.001$ \\
\hline Moderate & $0.62(0.42$ to 0.92$)$ & 0.016 & $0.43(0.34$ to 0.55$)$ & $<0.001$ & 0.47 (0.39 to 0.58$)$ & $<0.001$ \\
\hline Vigorous & $0.28(0.04$ to 2.00$)$ & 0.28 & 0.60 (0.31 to 1.17$)$ & 0.14 & 0.55 (0.29 to 1.03$)$ & 0.062 \\
\hline
\end{tabular}

${ }^{*}$ Adjustment factors: age, educational length, smoking, diabetes, previous myocardial infarction, previous stroke.P, significance level; RR, relative risk. 

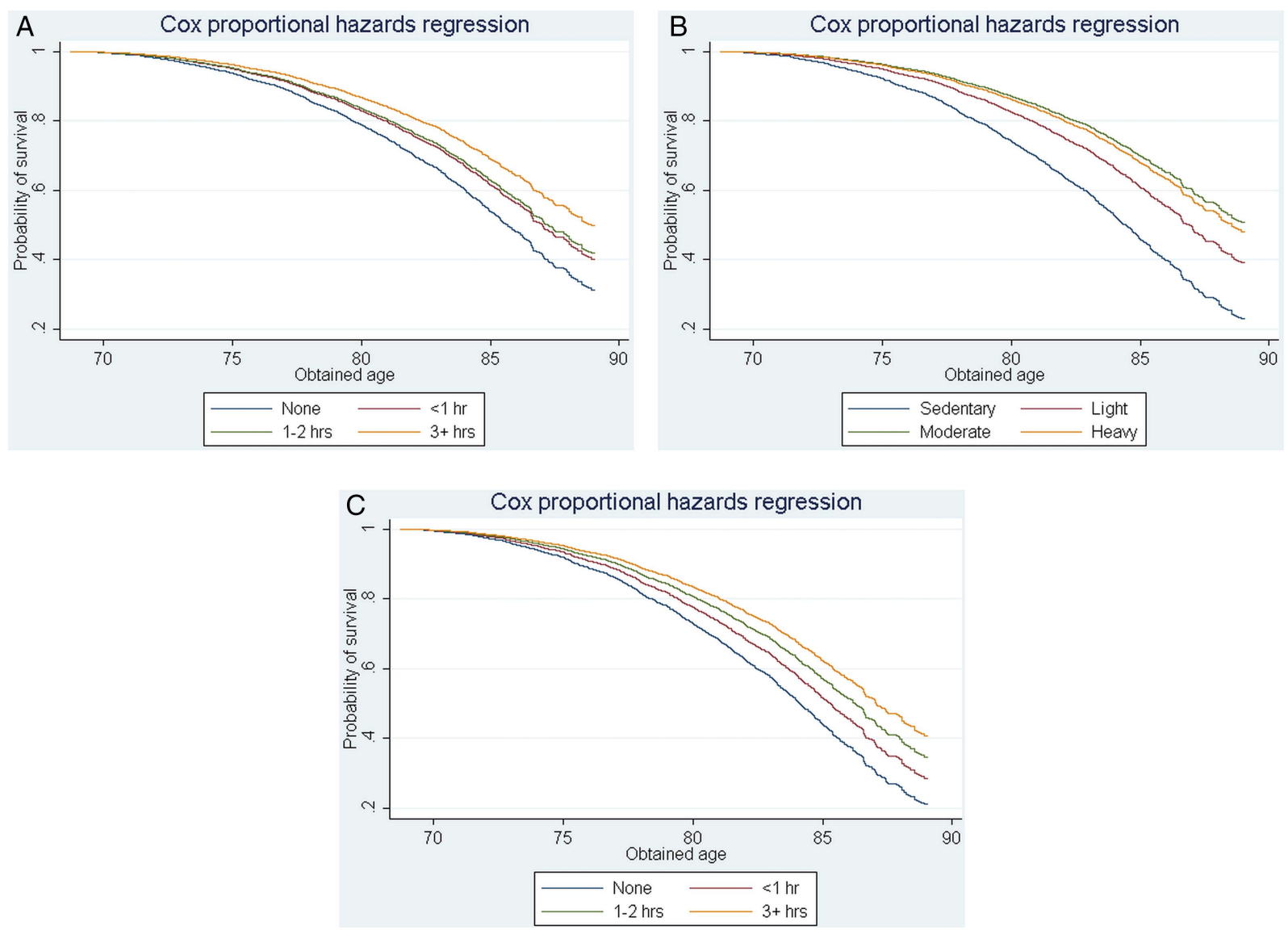

Figure 1 (A) Probability of survival until obtained age from year 2000 by amount of light intensity of physical activity per week, adjusted for educational length, smoking, diabetes, previous myocardial infarction and previous stroke. (B) Probability of survival until obtained age from year 2000 by amount of vigorous physical activity per week, adjusted for educational length, smoking, diabetes, previous myocardial infarction and previous stroke. (C) Probability of survival until obtained age from year 2000 by degree of physical activity at leisure, adjusted for educational length, smoking, diabetes, previous myocardial infarction and previous stroke.

of our exposure variables at leisure. Wen et $a l^{7}$ showed in a large study from Taiwan that $15 \mathrm{~min}$ of daily activity at a low intensity was associated with a $14 \%$ risk reduction through a 3 -year follow-up with a further dose-response reduction of $4 \%$ for each $15 \mathrm{~min}$ increased activity. This was somewhat weaker than observed in our data, but the authors adjusted for 13 variables (such as blood glucose and systolic blood pressure) that may have resulted in overadjustment.

The Gothenburg activity question showed a remarkably strong gradient with good ability to discriminate between persons with sedentary and intermediate/great degree of activity on median lifetime. Even when men were 73 years of age on average at start of follow-up, active persons had 5 years longer expected lifetime than the sedentary. This question measures type, amount and intensity of activity in a somewhat unstructured way, whereas the two intensity questions are not specific on type of activity. This could be part of the reason why the Gothenburg question outperformed the two intensity questions with regard to prognostic ability.

\section{Selection of men}

Men participating in Oslo I and II had 46\% (43-49\%) lower mortality risk than those participating in Oslo I only. It is plausible that non-attendants were more prevalent in those being sedentary and it is known from the Oslo I study mortality follow-up that non-attendants experienced higher mortality rates than attendants and that this was more pronounced in less educated than highly educated men. ${ }^{16}$ Thus, the large extended lifetime in the intermediate group of activity could even be an underestimate.

\section{Choice of adjustment factors}

There are more potential confounding factors than those adjusted for, such as serum glucose and lipoproteins. However, several of these could be a result of the physical activity level; so added adjustments for these could have led to overadjustments. In addition, Spearman correlation between degree of physical activity at leisure and at work was small $(r=0.058)$ in 1972/ 1973, when also the degree of physical activity at work was recorded. Thus, confounding of occupational differences in degree of activity at work was probably small in this study.

\section{Widespread prevention effects}

Historically, major emphasis has been put on the benefits to the CV system from physical activity. Also this study confirmed the strong and negative association between exposure activity variables and CV mortality. However, non-CV mortality was also strongly and negatively associated to all such variables. Thus, physical activity seems to affect many organ systems and may protect individuals from premature death even at an older age.

\section{Effect of competing risk}

The estimates of CV mortality according to exposure variables may have been biased due to a high degree of competing risk. 
Table 3 Relative risk of cardiovascular and non-cardiovascular mortality treating the other as a competing cause of death, by physical activity, adjusted for potential confounders*

\begin{tabular}{|c|c|c|}
\hline \multicolumn{3}{|l|}{ Cause of death } \\
\hline Physical activity & $\begin{array}{l}\text { Cardiovascular, competing } \\
\text { non-cardiovascular } \\
\text { RR }(95 \% \mathrm{CI})\end{array}$ & $\begin{array}{l}\text { Non-cardiovascular, } \\
\text { competing cardiovascular } \\
\text { RR }(95 \% \mathrm{CI})\end{array}$ \\
\hline \multicolumn{3}{|c|}{ Light intensity ( $\mathrm{N}=4662$ ) (hours) } \\
\hline None & 1 & 1 \\
\hline$<1$ & $0.90(0.50$ to 1.61$)$ & $0.81(0.54$ to 1.22$)$ \\
\hline $1-2$ & $0.71(0.44$ to 1.13$)$ & $0.76(0.55$ to 1.04$)$ \\
\hline $3+$ & $0.54(0.34$ to 0.86$)$ & $0.68(0.50$ to 0.93$)$ \\
\hline \multicolumn{3}{|c|}{ Hard intensity ( $\mathrm{N}=4543$ ) (hours) } \\
\hline None & 1 & 1 \\
\hline$<1$ & $0.63(0.45$ to 0.88$)$ & $0.87(0.73$ to 1.03$)$ \\
\hline $1-2$ & $0.78(0.57$ to 1.08$)$ & 0.77 (0.64 to 0.93$)$ \\
\hline $3+$ & $0.66(0.44$ to 1.00$)$ & $0.62(0.49$ to 0.80$)$ \\
\hline \multicolumn{3}{|c|}{ Degree at leisure $(\mathrm{N}=3500)$} \\
\hline Sedentary & 1 & 1 \\
\hline Light & $0.83(0.63$ to 1.10$)$ & $0.61(0.52$ to 0.72$)$ \\
\hline Moderate & $0.63(0.40$ to 0.97$)$ & 0.45 (0.35 to 0.58$)$ \\
\hline Vigorous & 0.40 (0.05 to 3.01$)$ & $0.51(0.24$ to 1.08$)$ \\
\hline
\end{tabular}

In the standard Cox model of time to $\mathrm{CV}$ death, preceding deaths are usually censored. This precludes CV death from occurring, making the exposure time shorter than it would have been had death not occurred. As a result, the rate of CV death defined as the number of $\mathrm{CV}$ deaths divided by total exposure time will be biased upwards due to the smaller denominator, thus overestimating the probability of $\mathrm{CV}$ death occurring within a certain time. The bias increases if the absolute risk of non-CV death increases. This approach does not provide information on the risk of $\mathrm{CV}$ death in participants who die first by a non-CV death, but risk factor associations with CV death can be provided for all participants, irrespective of whether they experience a non-CV death or not. However, the model is dependent on the assumption that the two end points are independent before either CV or non-CV death occurs, after adjustment for risk factors included in the model. Unfortunately, this assumption cannot be tested. The effect of competing risk will depend on the degree of competition by non-CV death, and it becomes progressively more important as absolute risk of non-CV death increases. Over and above these considerations, associations with risk factors may differ when comparing a standard and a competing risk Cox model, since the magnitude and direction of differences may vary according to the observed data, depending on how these factors are associated with both end points. However, a model including competing risk will probably give a better description of what is actually happening. In our situation, especially when analysing $\mathrm{CV}$ death, the competing risk model may give a more accurate risk estimate than the traditional model. The competing risk model estimated somewhat weaker gradients than the ordinary Cox regression model, more for the $\mathrm{CV}$ than for the non-CV end point. However, the main impression of a clear dose-response relationship for each exposure variable and each end point remained on the overall unchanged. In the physical activity versus mortality literature there are a few reports taking competing risk into account, generally for the younger participants: two regarding dose-response relationships between degree of walking and CV mortality, and other causes, ${ }^{17} 18$ and one in women. ${ }^{19}$ In these studies, total mortality was considerably lower than in our study and thus, degree of competing causes was also reduced. However, these confirmed the dose-response relationship between degree of physical activity and CV mortality.

\section{Predictive ability}

In the ROC analysis of risk comparison between physical activity and smoking measured in year 2000, the Gothenburg question variable was almost as predictive as smoking despite adjusting for the activity intensity variable. However, by collectively using both activity question variables, these performed better than the singular smoking question variable.

Increase in degree of physical activity at leisure had about equal predictive values as smoking cessation on all-cause mortality; this has also been found in a Swedish study. ${ }^{20}$ Such comparisons are obviously conditional on the prevalence of smoking and the various levels of activity. In year 2000, the prevalence of daily smoking was $20 \%$ among those who attended both studies. Since smoking behaviour has diminished to a large degree during the last decade also in Norway, it is reasonable to believe that the smoking prevalence now is more at the level of $15 \%$ in this age group. Thus, physical activity should be targeted to the same extent as smoking with respect to public health prevention efforts in the elderly.

\section{Study limitations}

Only the healthiest participants of the Oslo I cohort attended the Oslo II study. Absolute risk levels were, therefore, biased downwards, but adjusted relative risks between levels of activity variables as well as added life times by increased activity may

Table 4 ROC area $(95 \% \mathrm{Cl})$ for cardiovascular, non-cardiovascular and all-cause mortality in different models comparing predictive value of physical activity versus smoking, adjusted*

\begin{tabular}{|c|c|c|c|}
\hline Model & CV mortality $(n=310)$ & Non-CV mortality $(n=1064)$ & All-cause mortality $(\mathrm{N}=1374)$ \\
\hline $\mathrm{AF}$ & $0.725(0.684$ to 0.755$)$ & $0.650(0.631$ to 0.670$)$ & $0.699(0.682$ to 0.717$)$ \\
\hline AF except smoking & $0.720(0.689$ to 0.750$)$ & $0.636(0.616$ to 0.656$)$ & $0.686(0.668$ to 0.717$)$ \\
\hline AF except activity intensity & $0.720(0.690$ to 0.749$)$ & $0.650(0.631$ to 0.670$)$ & 0.699 (0.682 to 0.717$)$ \\
\hline AF except Gothenburg & $0.723(0.692$ to 0.753$)$ & $0.636(0.616$ to 0.656$)$ & $0.689(0.671$ to 0.707$)$ \\
\hline AF except Gothenburg and activity intensity & $0.715(0.686$ to 0.745$)$ & $0.628(0.608$ to 0.647$)$ & $0.680(0.662$ to 0.697$)$ \\
\hline AF except smoking, Gothenburg and activity intensity & $0.698(0.667$ to 0.728$)$ & $0.600(0.580$ to 0.620$)$ & $0.654(0.631$ to 0.673$)$ \\
\hline
\end{tabular}


have been less biased. Also self-reported activity levels may have been biased and probably in the direction of reporting more activity than actually performed in line with comparison studies between self-reported and objectively measured physical activity. ${ }^{21}$ The set of adjustment factors used here was selected from previous studies and these are known to be associated both with mortality and physical activity.

\section{CONCLUSIONS}

There was a strong and negative dose-response relationship between all physical activity exposure variables and CV, non-CV and all-cause mortality. A mortality reduction of $40 \%$ was associated with a moderate use of time ( $30 \mathrm{~min} 6$ days a week) irrespective of whether the activity was light or vigorous. Modelling by competing risk of non-CV death on CV death and vice versa weakened associations to exposure variables to a certain degree, but associations were still significant. Increased physical activity was as beneficial as smoking cessation in reducing all-cause mortality. Public health strategies to reduce risk in elderly men should concentrate highly on promoting increased physical activity in their action plans.

\section{What are the new findings?}

- In elderly men, increased physical activity is as important for survival as quitting smoking.

- Both cardiovascular (CV) as well as non-CV mortality are reduced by increasing the degree of physical activity in a dose-response pattern.

- Accounting for non-CV mortality in a competing risk model when analysing CV mortality or vice versa showed a slightly weakened relationship, but the interpretation did not change.

- Thirty minutes of moderate activity 6 days a week was associated with $40 \%$ mortality risk reduction.

\section{How might it impact on clinical practice in the near future?}

- More time and resources should be allocated in primary care to increase the degree of physical activity among the elderly.

- Equally more time and resources should be used to advice on smoking cessation as well as increased degree of physical activity in the elderly.

- Physicians should emphasise the broad spectrum of diseases and non-CV causes of death that can be prevented by an increased degree of physical activity in the elderly.

Contributors $\mathrm{IH}$ made all statistical analyses for the mortality study and wrote the first draft. SAA made major revisions to the mortality study and contributed by a major extent to all sport scientific considerations.

Competing interests None declared.

Patient consent Obtained.
Ethics approval South Eastern Region Health Ethical Committee.

Provenance and peer review Not commissioned; externally peer reviewed.

Data sharing statement The data for the Oslo study are stored at the Norwegian Institute of Public Health in Oslo, Norway. All scientists may apply to use the data for getting solutions for their interesting scientific questions.

\section{REFERENCES}

1 Lee IM, Shiroma EJ, Lobelo F, et al. Effect of physical inactivity on major non-communicable diseases worldwide: an analysis of burden of disease and life expectancy. Lancet 2012;380:219-29.

2 Lollgen H, Bockenhoff A, Kapp G. Physical activity and all-cause mortality; an updated meta analysis with different intensity categories. Int J Sports Med 2009:30:213-24.

3 Bijnen FC, Caspersen CJ, Feskens EJ, et al. Physical activity and 10-year mortality from cardiovascular diseases and all causes: the Zutphen Elderly Study. Arch Intern Med 1998;158:1499-505.

4 Holme I, Helgeland A, Hjermann I, et al. Physical activity at work and at leisure in relation to coronary risk factors and social class. A 4-year mortality follow-up. The Oslo study. Acta Med Scand 1981;209:277-83.

5 Wisloff U, Nilsen TI, Droyvold WB, et al. A single weekly bout of exercise may reduce cardiovascular mortality: how little pain for cardiac gain? 'The HUNT study, Norway'. Eur J Cardiovasc Prev Rehabil 2006;13:798-804.

6 Almeida OP, Kahn KM, Hnakey G, et al. 150 minutes of vigorous physical activity per week predicts survival and successful ageing: a population-based 11-year longitudinal study of 12201 older Australian men. Br J Sports Med 2014:48:220-5.

7 Wen CP, Wai JP, Tsai MK, et al. Minimum amount of physical activity for reduced mortality and extended life expectancy: a prospective cohort study. Lancet 2011;378:1244-53.

8 Yusuf $\mathrm{S}$, Hawken $\mathrm{S}$, Ounpuu $\mathrm{S}$, et al. Effect of potentially modifiable risk factors associated with myocardial infarction in 52 countries (the INTERHEART study): case-control study. Lancet 2004;364:937-52.

9 Leren P, Askevold EM, Foss OP, et al. The Oslo Study, cardiovascular disease in middle-aged and young Oslo men. Acta Med Scand 1975;588 (Suppl):1-38.

10 Holme I, Tonstad S. Association of coronary heart disease mortality with risk factors according to length of follow-up and serum cholesterol level in men: the Oslo Study cohort. Eur J Prev Cardiol 2013;20:168-75.

11 Holme I, Tonstad S, Sogaard AJ, et al. Leisure time physical activity in middle age predicts the metabolic syndrome in old age: results of a 28-year follow-up of men in the Oslo study. BMC Public Health 2007;7:154.

12 Saltin B, Grimby G. Physiological analysis of middle-aged and old former athletes. Circulation 1968;38:1104-15.

13 Aires N, Selmer R, Thelle D. The validity of self-reported leisure time physical activity, and its relationship to serum cholesterol, blood pressure and body mass index. A population based study of 332,182 men and women aged 40-42 years. Eur J Epidemiol 2003;18:479-85.

14 Fine JP, Gray RJ. A proportional hazards model for the subdistribution of a competing risk. J Am Stat Assoc 1999;94:496-509.

15 StataCorp.2011. Statrelease 12. Statistical software. College Station, TX: StataCorp LP, 2011.

16 Holme I. Coronary risk factors and their possible causal role in the development of coronary heart disease. Thesis. Norway: University of Oslo, 1982.

17 Williams PT, Thompson PD. The relationship of walking intensity to total and cause-specific mortality. Results from the National Walkers'Health Study. PLoS ONE 2013;8:e81098.

18 Williams PT. Dose-response relationship of physical activity to premature and total all-cause and cardiovascular disease mortality in walkers. PLOS ONE 2013;8: e78777.

19 Baer HJ, Glynn RJ, Hu FB, et al. Risk factors for mortality in the nurses' healthy study: a competing risks analysis. Am J Epidemiol 2011;173:319-29.

20 Byberg L, Melhus H, Gedeborg R, et al. Total mortality after changes in leisure time physical activity in 50-year-old men: 35 year follow-up of population based cohort. BMJ 2009;338:b688.

21 Dyrstad SM, Hansen BH, Holme IM, et al. Comparison of self-reported versus accelerometer-measured physical activity. Med Sci Sports Exerc 2014;46:99-106. 